\title{
Propofol attenuated liver transplantation-induced acute lung injury via connexin43 gap junction inhibition
}

Dongdong Yuan ${ }^{\dagger}$, Guangjie Su$^{\dagger}$, Yue Liu ${ }^{\dagger}$, Xinjin Chi, Jiayu Feng, Qianqian Zhu, Jun Cai ${ }^{*}$, Gangjian Luo* and Ziqing $\mathrm{Hei}^{*}$

\begin{abstract}
Background: Postoperative acute lung injury (ALI) is a severe complication after liver transplantation, which influences patient survival rate obviously. However, its mechanisms are unclear and effective therapies are still lacking. The current study focused on effects of propofol on liver transplantation-induced ALI and whether its underlying mechanism was relative with connexin43 (Cx43) alternation. The authors postulated that endotoxin induced enhancement of $C \times 43$ gap junction (GJ) plays a critical role in mediating post liver transplantation ALI and that pretreatment with the anesthetic propofol, known to inhibit gap junction, can confer effective protection.
\end{abstract}

Methods: Male Sprague-Dawley rats underwent autologous orthotopic liver transplantation (AOLT) in the absence or presence of treatments with the selective Cx43 inhibitor, enanthol $(0.1 \mathrm{mg} / \mathrm{kg})$ and propofol $(50 \mathrm{mg} / \mathrm{kg})$, a commonly used anesthetic in clinical anesthesia. In vitro study, BEAS-2B cells, a kind of lung epithelial cell line expressing Cx43, exposed to lipopolysaccharide (LPS), which mainly contributed to ALI. Function of CX43 GJ was regulated by Cx43 specific inhibitors, gap26 (300 $\mu \mathrm{M})$ or enhancer, retinoic acid $(10 \mu \mathrm{M})$ and two specific siRNAs.

Results: Compared with the sham group, AOLT results in ALI obviously with plasma endotoxin increase. CX43 inhibition decreased ALI through inflammatory reaction reduction. In vitro studies, LPS-induced BEAS-2B cells damage was attenuated by $\mathrm{C} \times 43$ function inhibition, but amplified by enhancement. Another important finding was propofol reduced CX43 function and protected against LPS-mediated BEAS-2B cells damage or AOLT-induced ALI, mechanisms of which were also associated with inflammatory reaction decrease.

Conclusion: Cx43 plays a vital role in liver transplantation-induced ALI. Propofol decreased Cx43 function and protected against ALI in vivo and in vitro. This finding provide a new basis for targeted intervention of organ protection in liver transplantation, even in other kinds of operations.

Keywords: Liver transplantation, Acute lung injury, Connexin43, Propofol

\section{Background}

Liver transplantation is always considered to be the most effective and efficient therapy for patients to cure the end-stage liver disease [1]. However, various kinds of postoperative complications affect patient survival

\footnotetext{
*Correspondence: gzcaijun@hotmail.com; 18922102871@163.com; heiziqing@hotmail.com; heiziqing@sina.com

${ }^{\dagger}$ Dongdong Yuan, Guangjie Su and Yue Liu contributed equally to this study

Department of Anesthesiology, The Third Affiliated Hospital of Sun Yatsen University, Tianhe Road, Guangzhou, People's Republic of China
}

obviously, one of which is just ALI [2]. Causes of ALI is very complex and involve multiple factors, among of which enterogenous endotoxin over-production with its associated inflammatory cytokines explosion is considered to play an important role in this process $[3,4]$. During liver transplantation, because inferior vena cava and the portal vein are interrupted, intestinal congestion becomes obvious, which results in intestine motility and barriers destroyed significantly. Both bacterial translocation and enterogenous endotoxin are over-produced, leading to susceptible organs injuries, including lungs [5, 
6]. As reported, ALI contributed to mortality of patients suffering from liver transplantation, because patients with ALI prone to develop acute respiratory distress syndrome (ARDS), mortality rate among of which could be as high as $76.5 \%[6,7]$. However, mechanisms of this complication are still unclear and effective therapies are lacking. Thus, therapeutic strategies investigation about liver transplantation-induced ALI becomes a new focus in recent years, which is beneficial for patients' recovery.

As transmembrane proteins, connexins express widely in different kinds of organs and tissues. This big family contains about 21 isoforms and six connexin monomers form a hemichannel on the neighboring cells docking together to form an integral gap junction channel, called gap junction (GJ). Molecule weight of signals less than $1 \mathrm{kDa}$ could be transferred through this special channels, including calcium, cyclic adenosine monophosphate, cyclic guanosine monophosphate, glutathione, etc. Direct signal transfer mediated by GJ always plays an important part in physiological and pathological events $[8,9]$. Cx43 (molecular weight is $43 \mathrm{kDa}$ ) is one of the most important connexins as far as we know and it is always relative with ALI $[10,11]$. As reported, Cx43 and its function alternation could affect the inflammatory factors secretion and intercellular communication, which was involved in the on come and development of pulmonary inflammation and lung edema [12]; others demonstrated that $\mathrm{Cx} 43$ mediated spread of $\mathrm{Ca}^{2+}$-dependent proinflammatory responses in lung capillaries [13]. Results above provide a clue that Cx43 is important for lung injury, but function of it on liver transplantation-induced ALI is still unknown. That is one of the key point in our investigation.

Propofol, as a commonly used anesthetic in clinical anesthesia, is considered to have positive effects on organ protection, which has been explored for many years, however, its underlying mechanism is still unclear [1416]. In our previous studies, we had demonstrated that propofol could attenuate liver transplantation-induced acute kidney injury or decrease X-ray induced cellular toxicity through inhibiting GJ composed of Cx32 [9, 17]. Based on these findings, we hypothesized protective effects of propofol were relative with GJ mediation. Thus, in current study, we investigate protective effects of propofol on liver transplantation-induced ALI and whether its underlying mechanism is relative with $\mathrm{Cx} 43$ function inhibition. This investigation could confer a new basis for therapy development to combat liver transplantation-mediated ALI.

\section{Methods}

Animals and treatment

Male Sprague-Dawley rats (200-220 g) were purchased from Sun Yat-Sen University. The study was approved by the Laboratory Animal Care Committee of Sun Yat-Sen University (Guangzhou, Guangdong, China) and animal care followed National Institutes of Health criteria for the care and use of laboratory animals in research. Enanthol (Sigma-Aldrich, St. Louis, MO) was given at $0.1 \mathrm{mg} /$ $\mathrm{kg} 4 \mathrm{~h}$ before AOLT and propofol (Sigma-Aldrich, St. Louis, MO) was given at $50 \mathrm{mg} / \mathrm{kg}$ for 3 days intraperitoneally before AOLT. This dosage of propofol has been widely used in in vivo studies in rats and produced beneficial effects. Our previous studies showed that propofol $50 \mathrm{mg} / \mathrm{kg}$, produced a sedative response in rats, as determined by loss of reflex responses to a painful stimulus while remaining sensitive to skin incision [9].

AOLT models of rat were established according to our previous study. Rats were fasted for $8 \mathrm{~h}$ with free access to drinking water prior to surgery. Open face guard was used to administer the inhalational ether anesthesia until rats did not have response to a needle stimulus. Subsequent to entering the abdominal cavity, the falciform ligament of the liver was resected and ligated, and the left vena phrenica along the esophagus was severed. The liver was revealed until the supra hepatic vena cava (SVC) was liberated and then, and then placed back into its original position. A bold line was prepared to guide the SVC for blockage easily using vascular clamps at a later stage. After left renal vein upper region was liberated, we dissociated the inferior vena cava (IVC), dissected the first hepatic portal and separated the portal vein (PV) from the convergence of the inferior mesenteric and splenic veins. And then hepatic artery and biliary were both liberated according to their anatomic relationship. Subsequently, we ligated the portal hepatics and used microvascular clamps at the convergence of the inferior mesenteric, splenic veins, hepatic artery, SVC, and IVC. The PV was punctured with a 24-gauge needle for reperfusion. We made one 1-mm incision on the IVC wall as an outflow and injected $4{ }^{\circ} \mathrm{C}$ Ringer lactate solution at $2.5 \mathrm{ml} / \mathrm{min}$ until the liver color turned yellow and closed the opening of the PV and IVC with 8-0 sutures. PV, SVC, IVC, and hepatic artery were all unclamped. The anhepatic phase lasted for $20 \pm 1 \mathrm{~min}$ on average $[5,9$, 14].

\section{Assessment of lung damage}

Eight hours after AOLT, $2 \mathrm{ml}$ air was injected into the tail vein in order to sacrifice the rats. Lung tissues were removed. The middle lobe of the right lung was weighed on an electronic scale and the inferior lobe was fixed in $10 \%$ buffered formalin, embedded in paraffin for hematoxylin-eosin staining. According to the scores of Franco-Gou et al., the pathology was analyzed blindly. The graded edema of the alveolar mesenchyme, intraalveolar cell infiltration and alveolar hemorrhage were 
scored. We measured the wet weight of the superior lobe of the right lung and placed it in an oven for $24 \mathrm{~h}$ at $80{ }^{\circ} \mathrm{C}$ in order to evaporate. The water content of the lung was calculated as: Water content $=$ (lung wet weight-lung dry weight)/lung wet weight $\times 100[18,19]$.

\section{Detection of LPS, tumor necrosis factor- $\alpha$ (TNF- $\alpha$ ),} interleukin-1 $\beta$ (IL-1 $\beta$ ), interleukin-6 (IL-6) and interleukin-8 (IL-8)

LPS, TNF- $\alpha$, IL- $1 \beta$, IL- 6 , and IL- 8 are determined by their own assay kits following introductions (SigmaAldrich). We standardized the results of cytokines (relative LPS, TNF- $\alpha$, IL-1 $\beta$, IL-6, and IL-8) of the experimental sham group in vivo or control group in vitro as 1 , and the values of other groups were presented as relative values compared to experimental sham group in vivo or control group in vitro, with the purpose to easily visualize the changes of cytokines (relative LPS, TNF- $\alpha$, IL- $1 \beta$, IL-6, and IL-8) resulted from changes of Cx43.

\section{Western blotting}

Western blotting follows the standard procedures as previous studies [20]. Cx43 expression were detected with anti-Cx43 (Sigma-Aldrich; 1:4000) and secondary antibody (Sigma-Aldrich; 1:5000). Anti- $\beta$-actin and its corresponding secondary antibody (Sigma-Aldrich) were at 1:4000 [21].

\section{Immunohistochemical staining}

According to the appropriate protocol, immunohistochemical staining was performed in $4 \mu \mathrm{m}$ paraffinized sections. After being dewaxed and dehydrated, the sections were incubated with $3 \% \mathrm{H}_{2} \mathrm{O}_{2}$, inhibiting endogenous peroxidase activity. The primary antibodies against Cx43 was 1:1000 (Sigma-Aldrich) for $24 \mathrm{~h}$ at $4{ }^{\circ} \mathrm{C}$. After incubated with its corresponding secondary antibody (Sigma-Aldrich; 1:4000), samples were observed with a light microscope (EclipseE800, Nikon, Tokyo, Japan) [9].

\section{Cell culture and treatments}

BEAS-2B cells, a kind of lung epithelial cell line was obtained from American Type Culture Collection (Manassas, VA, USA). Cells were grown at $37^{\circ} \mathrm{C}$ in an atmosphere of $5 \% \mathrm{CO}_{2}$ in air and cultured in DMEM/F-12 supplemented with $10 \%$ fetal bovine serum. BEAS-2B cells were pretreated with connexin channel inhibitors gap26, $300 \mu \mathrm{M}$, for $1 \mathrm{~h}$ (Sigma-Aldrich, a connexinmimetic peptide) and a $\mathrm{Cx} 43$ expression enhancer, retinoic acid (RA) $10 \mu \mathrm{M}$, for $24 \mathrm{~h}$ (Sigma-Aldrich) before LPS (Sigma-Aldrich, $5 \mu \mathrm{g} / \mathrm{ml}, 24 \mathrm{~h}$ ) treatment. Corresponding solvents of gap26 and RA are DMSO [22, 23].

Propofol was at the concentration of $15 \mu \mathrm{M}$ for $1 \mathrm{~h}$ before being subjected to LPS exposure. This concentration at $15 \mu \mathrm{M}$ was based on our investigation which showed propofol at $15 \mu \mathrm{M}$ can profoundly decrease dye coupling and also based on our previous study. This concentration of propofol $(15 \mu \mathrm{M})$ is in the range of target plasma concentration of propofol 2-4 $\mu \mathrm{g} /$ $\mathrm{ml}$ (i.e., 11-22 $\mu \mathrm{M}$ ) as used clinically during major surgeries $[24,25]$.

\section{"Parachute" dye-coupling assay}

"Parachute" dye-coupling assay was used to detected functional GJ as described $[8,9]$.

\section{Cell counting kit-8 (CCK8) and LDH release assay}

Cells growth were determined in 24-well plates with CCK8 assay, which was carried out according to the introduction (Dojindo, Tokyo, Japan). LDH release were detected in 96-well plates with its corresponding kits (Sigma-Aldrich) [26].

\section{Cx43 knock-down with small interfering RNA (siRNA) transfection}

Two specific siRNAs (GCTGGTTACTGGTGACAGA and CCGCAATTACAACAAGCAA, Cx43-siRNA1 and Cx43-siRNA2) targeting Cx43 gene were used to reduced Cx43 expression. A nonspecific Cx43-siRNA (NC as shown in the Fig. 4) was used as controls. Lipofectamine 2000 (Invitrogen, Carlsbad, CA, USA) was used to transfect siRNA according to the manufacturer's instructions.

\section{Statistical analysis}

Statistical analysis was performed with SPSS 15.0 software (SPSS Inc., Chicago, IL). Multiple comparisons were analyzed with repeated measures one-way ANOVA, which is followed by Tukey post hoc comparisons. A two-tailed $\mathrm{P}$ value less than 0.05 was considered statistically significantly different.

\section{Results \\ Inhibition GJ composed of Cx43 alleviated ALI followed AOLT}

Rat AOLT model was established to explore effects of liver transplantation on lungs. ALI was most serious at $8 \mathrm{~h}$ after AOLT which was the same as our previous study [5]. In AOLT model group, alveolar exudates and inflammatory cell infiltration were obvious; pulmonary interstitium exhibited significant hyperemia and severe hemorrhage. Pathological scores and water content were both higher than that in sham group. Enanthol, a kind of Cx43 inhibitor, alleviated ALI followed AOLT and both pathological scores and water content were improved obviously (Fig. 1a, b).

As reported, intestine motility and barriers were destroyed after liver transplantation, which lead to 


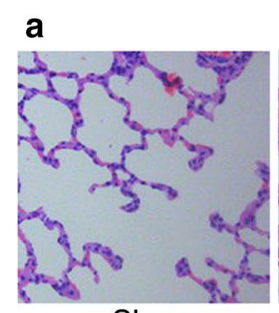

Sham

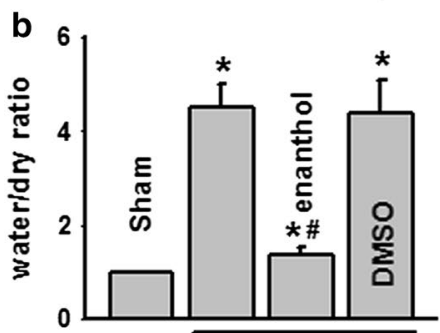

AOLT

e

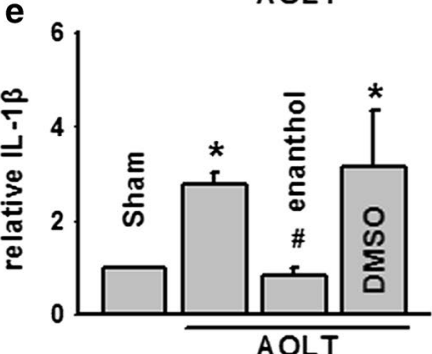

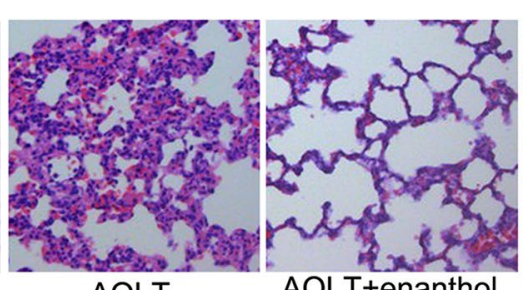

AOLT+enanthol

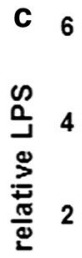

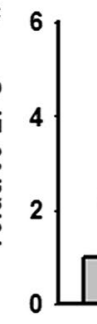

\section{$\mathbf{f}$}

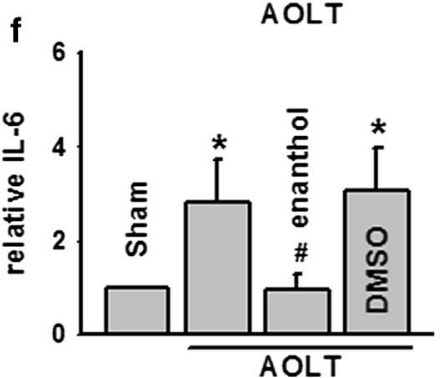

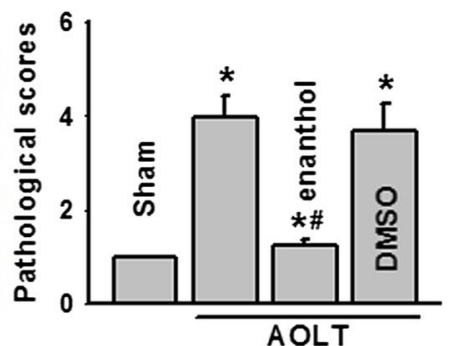
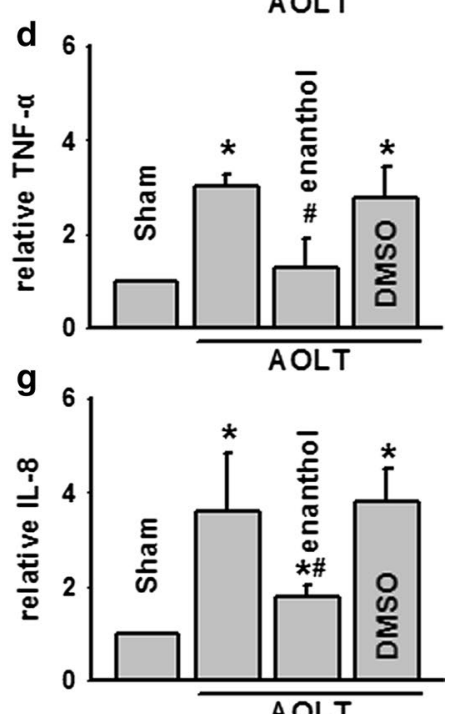

Fig. 1 AOLT-induced ALI was attenuated by enanthol application. Rats were exposed to enanthol $(0.1 \mathrm{mg} / \mathrm{kg})$ or DMSO for $1 \mathrm{~h}$ before AOLT and all samples were obtained $8 \mathrm{~h}$ after reperfusion. a Pathological damage of lungs ( $\mathrm{H} \& \mathrm{E}$ staining; original magnification $200 \times$ ). $\mathbf{b}$ Water content of the

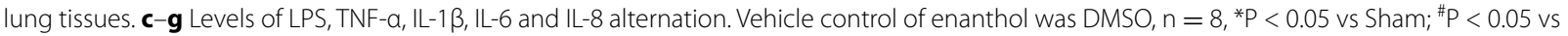
AOLT

bacterial translocation and enterogenous endotoxin explosion [5]. Inflammatory cytokines were over produced triggering organ injuries, among of which lungs were the most susceptible organs for endotoxin and inflammatory cytokines $[5,6]$. Thus, results in Fig. 1c to g showed that changes of LPS and cytokines were coincident with lung pathological damage after AOLT: LPS, TNF- $\alpha$, IL-1 $\beta$, IL- 6 and IL- 8 were all increased in AOLT model group, which could be reduced by enanthol pretreatment significantly. DMSO, as vehicle control of enanthol had no effects on the parameters.

\section{Enanthol alleviated lung Cx43 expression followed AOLT}

It has been reported that connexin over-expression always related with organ damage in our previous studies $[9,27]$. And results in Fig. 1 also provided a clue that Cx43 might play an important role in AOLT-mediated ALI because of the inhibitor of $\mathrm{Cx} 43$, enanthol pretreatment alleviating lung pathological damage and inflammatory cytokines explosion significantly. Thus, we have every reason to suppose that $\mathrm{Cx} 43$ expression played an important role in AOLT-mediated ALI. Two different methods, western blotting and immunohistochemisty were carried out to visualize expression and localization of $\mathrm{Cx} 43$, both of which showed that $\mathrm{Cx} 43$ expression was increased obviously after AOLT, but to be inhibited by the inhibitor of Cx43, enanthol, whereas DMSO, vehicle control of enanthol had no effects on Cx43 expression and localization (Fig. 2). These results were coincident with changes of pathological damage and cytokines explosion in Fig. 1, which supported our hypothesis that Cx43 inhibition attenuated ALI after AOLT via decreasing inflammatory reaction.

\section{Alternation of $\mathrm{Cx} 43$ function influenced LPS-induced BEAS- $2 B$ cells damage}

In order to confirm the hypothesis we have mentioned in vivo studies, we further tested it on BEAS-2B cells, a kind of lung epithelial cell line expressing Cx43 [28]. With the facts that endotoxin always played an important role in ALI after AOLT, and the destruction of intestine motility and barriers after liver transplantation lead to endotoxin explosion and inflammatory cytokines overproduction, resulting in susceptible organs injuries [29, 

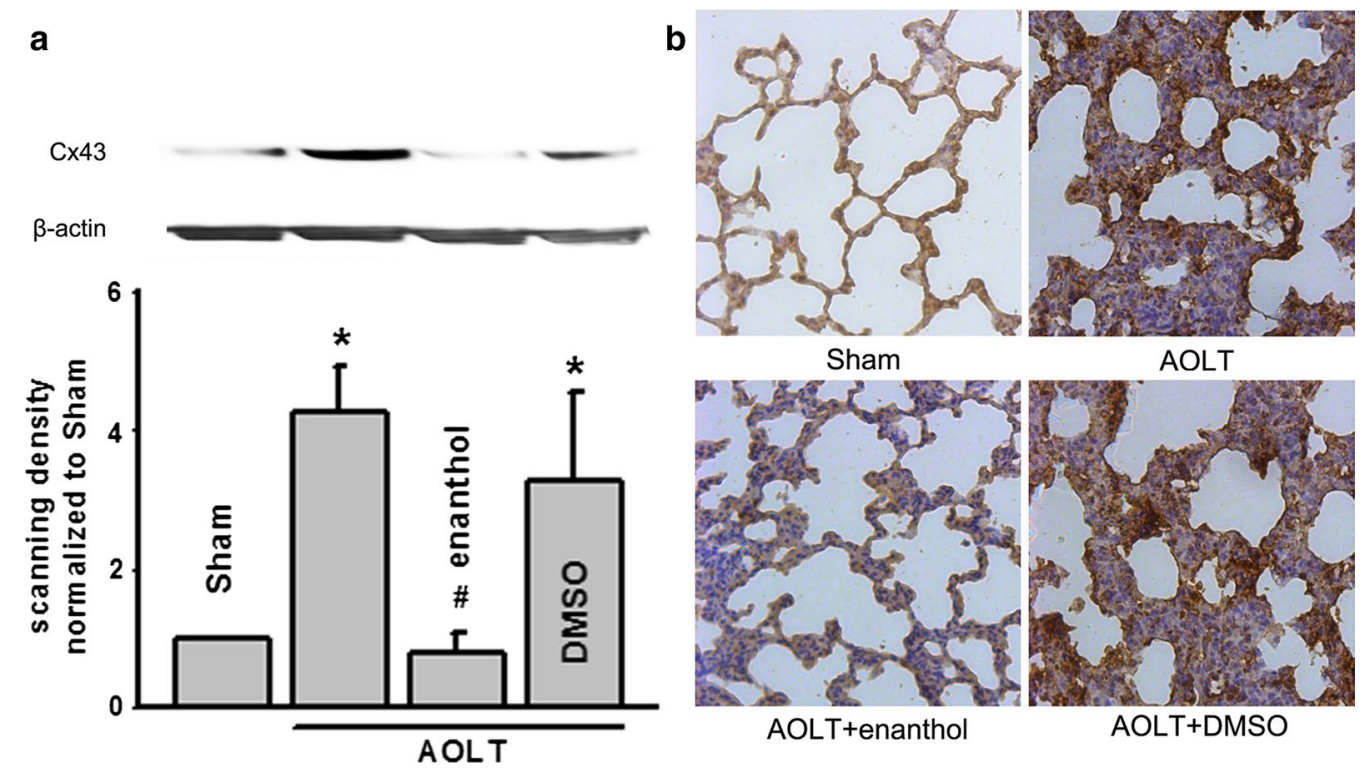

Fig. 2 AOLT-induced Cx43 expression increase was attenuated by enanthol application. Rats were exposed to enanthol (0.1 mg/kg) or DMSO for $1 \mathrm{~h}$ before AOLT and all samples were obtained $8 \mathrm{~h}$ after reperfusion. $\mathbf{a}$ Cx43 expression of lungs determined with western blotting. b Cx43 expression of lungs determined with immunohistochemisty. Vehicle control of enanthol was DMSO, $n=5$, ${ }^{*} P<0.05$ vs Sham; ${ }^{P}<<0.05$ vs AOLT

30], models of LPS-induced BEAS-2B cells were used to test protective effects of $\mathrm{Cx} 43$ inhibition. Dye coupling was reduced by gap26, a specific inhibitor of $\mathrm{Cx} 43$, but increased by the enhancer, retinoic acid (RA) (Fig. 3a). At the same time, LPS-induced cell damage was also reduced subsequent to pretreatment with gap26, but exacerbated after RA treatment. Changes in LDH release or other inflammatory cytokines were just opposed to the changes in cell growth. Following pre-incubation with gap26, LDH release, TNF- $\alpha$, IL-1 $\beta$, IL- 6 and IL- 8 were all declined obviously, but increased dramatically with RA pre-treatment. DMSO, vehicle control of gap26 and RA had no effects on the parameters (Fig. 3b-g). These findings suggested that $\mathrm{Cx} 43 \mathrm{GJ}$ played a key role in LPSinduced BEAS-2B cellular damage through mediating inflammatory reaction.

\section{Cx32 gene knock-down to inhibit Cx32 GJ function prevented LPS-induced BEAS-2B cellular damage} In order to confirm the impact of $\mathrm{Cx} 43$ function on LPSinduced BEAS-2B cellular damage, we designed two different siRNAs targeting to Cx43 (Cx43-siRNA1 and Cx43-siRNA2) to specifically knock-down Cx43 expression (Fig. 4a). Blocking Cx43 expression depressed dye coupling of BEAS-2B cells (Fig. 4b). Although Cx43 knock-down per se did not affect cell growth under control condition, it attenuated LPS-induced reduction of cell growth and reduced LDH release significantly (Fig. 4c, d). LPS-induced TNF- $\alpha$, IL-1 $\beta$, IL-6 and
IL-8 were all inhibited because of siRNAs pretreatment (Fig. 4e, h).

\section{Propofol attenuated LPS-induced BEAS-2B cells damage via Cx43 inhibition}

Propofol had already been explored for many years and considered to have protective effects on different organs through different mechanisms. In our previous study, we had demonstrated that propofol could attenuate ALI followed AOLT [5], however, whether its mechanism was associated with GJ composed of $\mathrm{Cx} 43$ was still unclear. We first examined the effects of propofol on dye coupling of BEAS-2B cells, expressing Cx43. Figure 5a showed that the clinically relevant anesthesia concentration, $15 \mu \mathrm{M}$ propofol decreased dye coupling of BEAS-2B cells significantly,LPS-induced cell damage was reduced subsequent to pretreatment with propofol, and otherwise LDH release, TNF- $\alpha$, IL-1 $\beta$, IL- 6 and IL- 8 were also declined obviously (Fig. $5 \mathrm{~b}-\mathrm{g}$ ). These results showed that propofol attenuated LPS-induced BEAS-2B cell damage through $\mathrm{Cx} 43$ GJ inhibition, mechanism of which was relative with inflammatory reaction reduction. Fat milk, vehicle control of propofol had no effects on the parameters (Fig. 5b-g).

\section{Propofol alleviated ALI followed AOLT via Cx43 inhibition}

Following our above demonstration in Fig. 5 that propofol attenuated LPS-induced BEAS-2B cells damage via Cx43 inhibition resulting in inflammatory reaction 
a
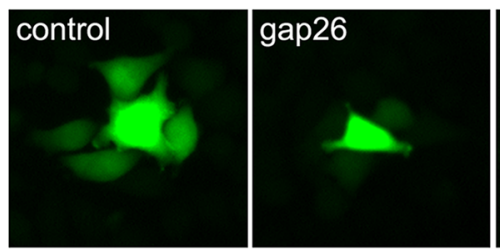

b
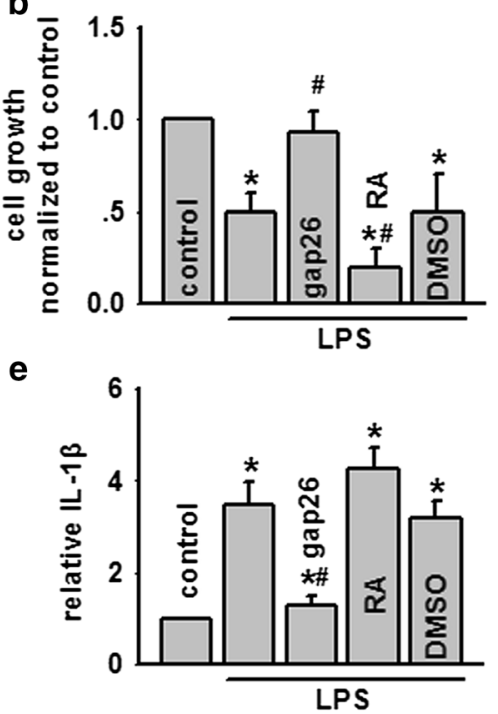

RA

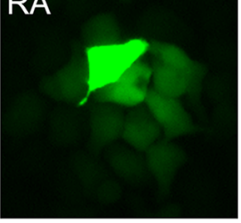

C

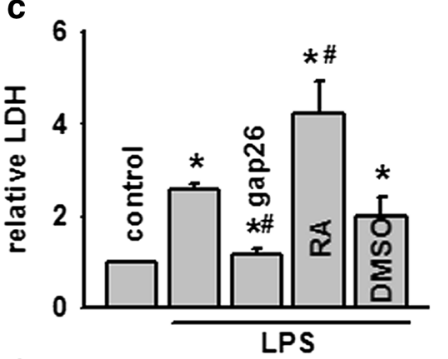

f

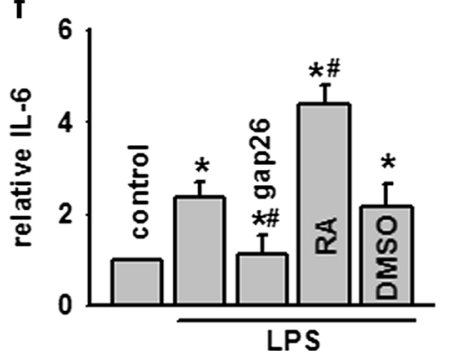

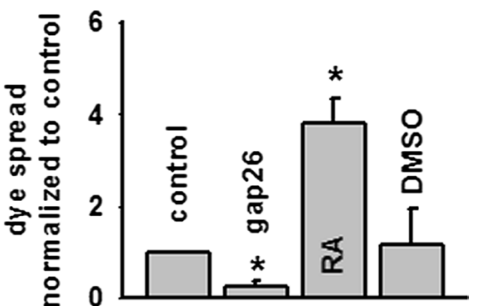

d
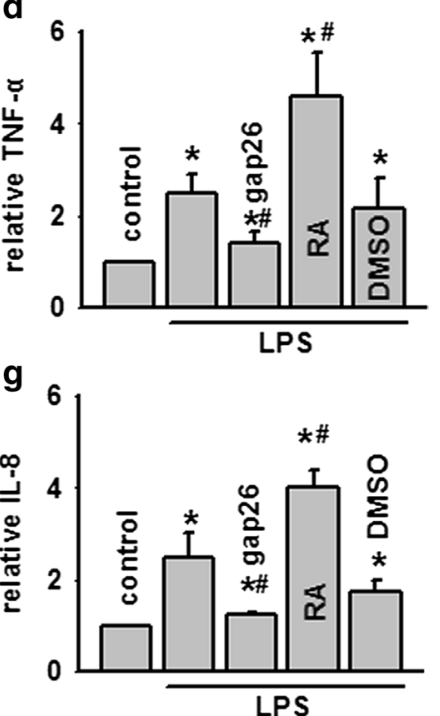

Fig. 3 LPS-induced BEAS-2B cells damage was attenuated by enanthol, but amplified by RA. a "Parachute" dye-coupling assay was used to deter-

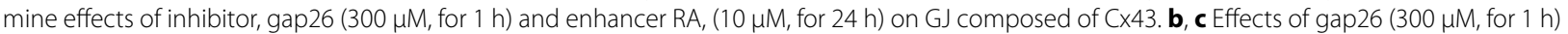
and RA $(10 \mu \mathrm{M}$, for $24 \mathrm{~h})$ on cell growth and LDH release exposed to LPS $(5 \mu \mathrm{g} / \mathrm{ml}$, for $24 \mathrm{~h}), \mathrm{n}=5$, ${ }^{*} \mathrm{P}<0.05 \mathrm{vs}$ control; ${ }^{\#} \mathrm{P}<0.05 \mathrm{vs}$ LPS. d- $\mathbf{g}$ Effects

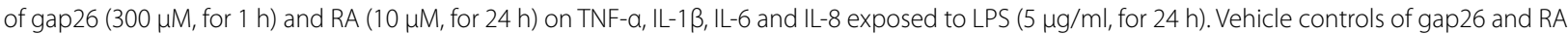
were DMSO, $n=5,{ }^{*} P<0.05$ vs control; ${ }^{*} P<0.05$ vs LPS

reduction, we wanted to further confirm the protective effects and mechanism of propofol in alleviating ALI followed AOLT in vivo. Both western blotting and immunohistochemisty showed that $\mathrm{Cx} 43$ expression was reduced significantly after propofol pretreatment (Fig. 6a, b), which was coincident with lung pathological damage (Fig. 6c, d). Results provided proof that propofol alleviated AOLT-induced ALI through Cx43 inhibition. More importantly, increase of LPS, TNF- $\alpha$, IL- $1 \beta$, IL- 6 and IL- 8 induced by AOLT was attenuated by propofol pretreatment obviously (Fig. 6e, i), whereas fat milk, vehicle control of propofol had no effects on the parameters.

\section{Discussion}

At present, liver transplantation is considered to be the most effective therapy to cure the final-stage liver disease [31]. However, ALI after liver transplantation is severe and influences patient survival significantly [32]. Until now, its underlying mechanism remains unclear. In present study, rat AOLT models were used to explore effects of $\mathrm{Cx} 43$ function on liver transplantation-induced
ALI, which closely mimicked the most procedure of liver transplantation, such as the SVC, IVC and PV blockade, intestine congestion, cold liver protection, fluid perfusion and liver ischemia reperfusion injury. This model had its own advantages. Compared with allogenic orthotopic liver transplantation, rat AOLT models avoided complex condition of recipients or reject reaction, kept good repeatability and high rat survival rate. From another aspects, rat AOLT model was better than the pure hepatic I/R injury model, because it not only included most procedures of liver transplantation, but also mimicked blood reflux disorder [9]. Thus, this kind of rat model was suitable for us to explore Cx43 GJ effects on ALI after liver transplantation and its potential therapeutic value. During liver transplantation, intestinal congestion was severe because of inferior vena cava and the portal vein interruption, resulting in intestine motility and barriers destroyed significantly. Bacterial translocation and enterogenous endotoxin over-production lead to susceptible organs injuries, such as lungs [33, 34]. It indicated that endotoxin played a central role in 

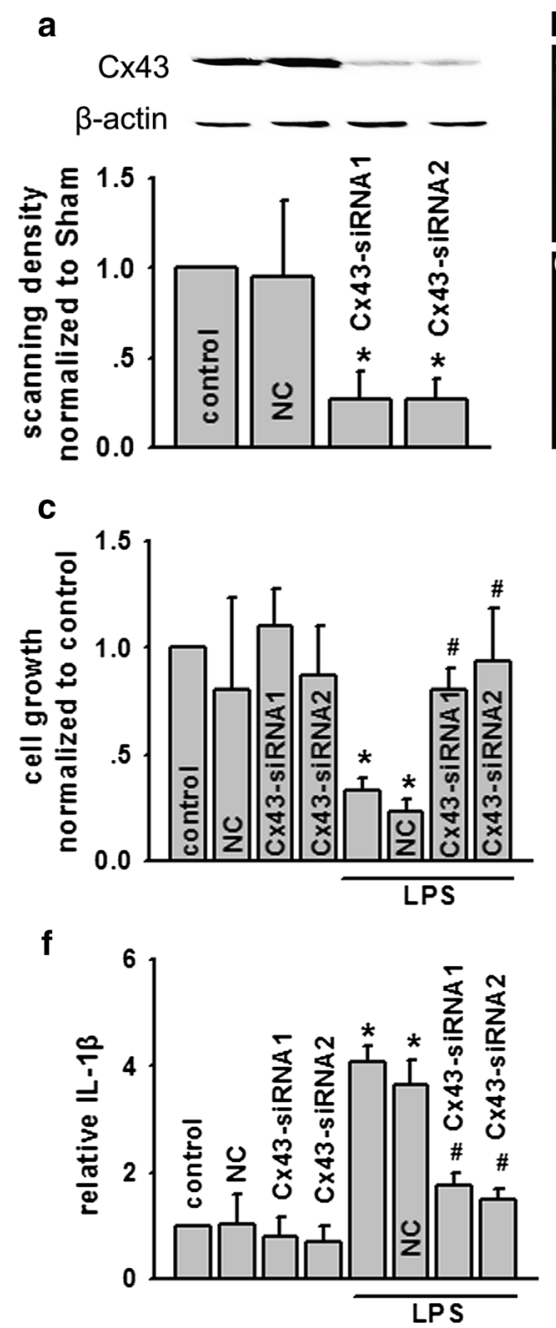
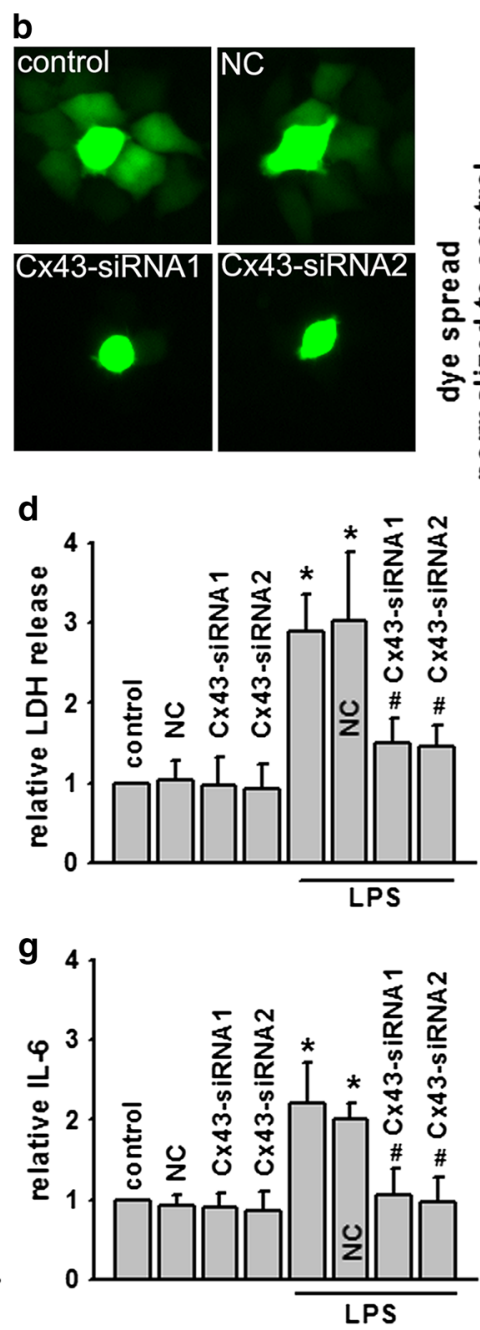
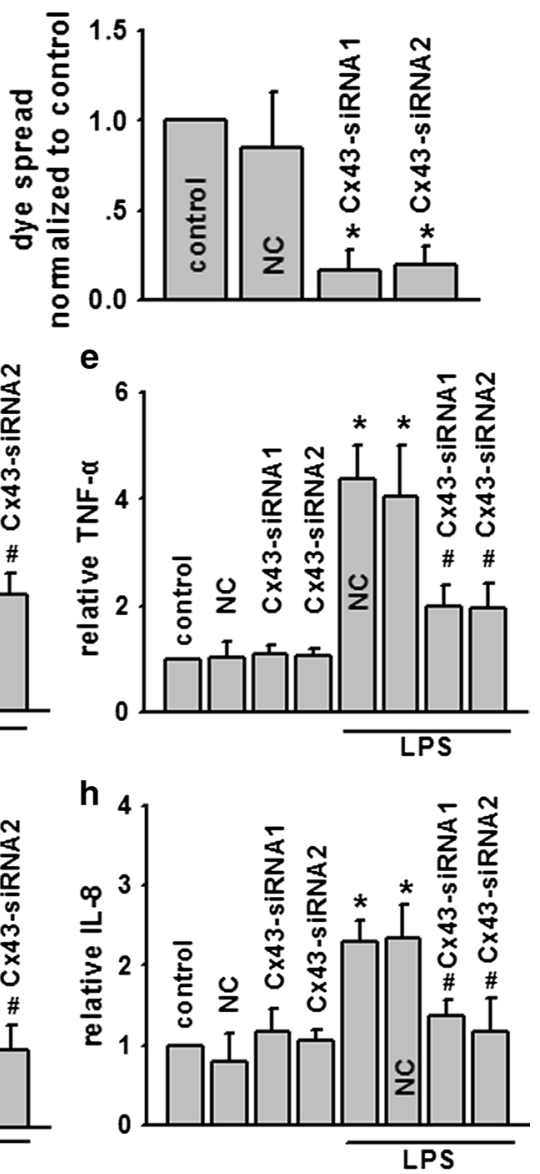

Fig. 4 SiRNA targeted Cx43 on BEAS-2B cells attenuated GJ function and LPS-induced cell damage. a, b Two different specific siRNAs decreased Cx43 expression dye-coupling of BEAS-2B cells. $\mathbf{c}$, $\mathbf{d}$ Effects of siRNAs on cell growth and LDH release exposed to LPS (5 $\mu \mathrm{g} / \mathrm{ml}, \mathrm{for} 24 \mathrm{~h}), \mathrm{n}=5$, ${ }^{*} \mathrm{P}<0.05$ vs control; ${ }^{\# P}<0.05$ vs LPS. e-h Effects of siRNA on TNF-a, IL-1 $\beta$, IL-6 and IL-8 exposed to LPS ( $5 \mu \mathrm{g} / \mathrm{ml}$, for $\left.24 \mathrm{~h}\right), \mathrm{n}=5$, *P $<0.05$ vs control; ${ }^{\#} \mathrm{P}<0.05$ vs LPS

liver transplantation-induced ALI. Thus, models of LPSinduced BEAS-2B cells, a kind of lung epithelial cell line expressing Cx43, were used to test protective effects of Cx43 inhibition in vitro.

On rat AOLT models, GJ composed of Cx43 inhibition alleviated ALI, which provided a clue that $\mathrm{Cx} 43$ function increase might be a major mechanism of liver transplantation-induced ALI. In vitro study, multiple manipulations regulating $\mathrm{Cx} 43$ function were used to further confirm this conclusion. Results showed that function of $\mathrm{Cx} 43 \mathrm{GJ}$ inhibition with specific inhibitor, gap26 or siRNA, alleviated LPS-induced BEAS-2B cells damage; by contrast, $\mathrm{Cx} 43$ GJ function increase with a selective enhancer, RA amplified this damage obviously, mechanisms of which were relative with inflammation reaction modulation. We also noticed that propofol, a common anesthetic used in clinic, protected against LPSinduced BEAS-2B cells damage through inhibiting Cx43 channels and that $\mathrm{Cx} 43$ inhibition was a major mechanism whereby propofol reduced inflammation reaction under pathological conditions like AOLT-induced ALI.

We had demonstrated that GJ composed of $\mathrm{Cx} 43$ played a vital role in AOLT-induced ALI. Others also reported that $\mathrm{Cx} 43$ mediated acid-induced increase in microvascular permeability and even regulated spread of $\mathrm{Ca}^{2+}$-dependent proinflammatory responses in lung capillaries [13]. GJ composed of connexins mediated direct cell-to-cell transfer of electrical charge or small molecules, which contributed to cell growth, physiology, or response to trauma $[35,36]$. Cell signals transferred 

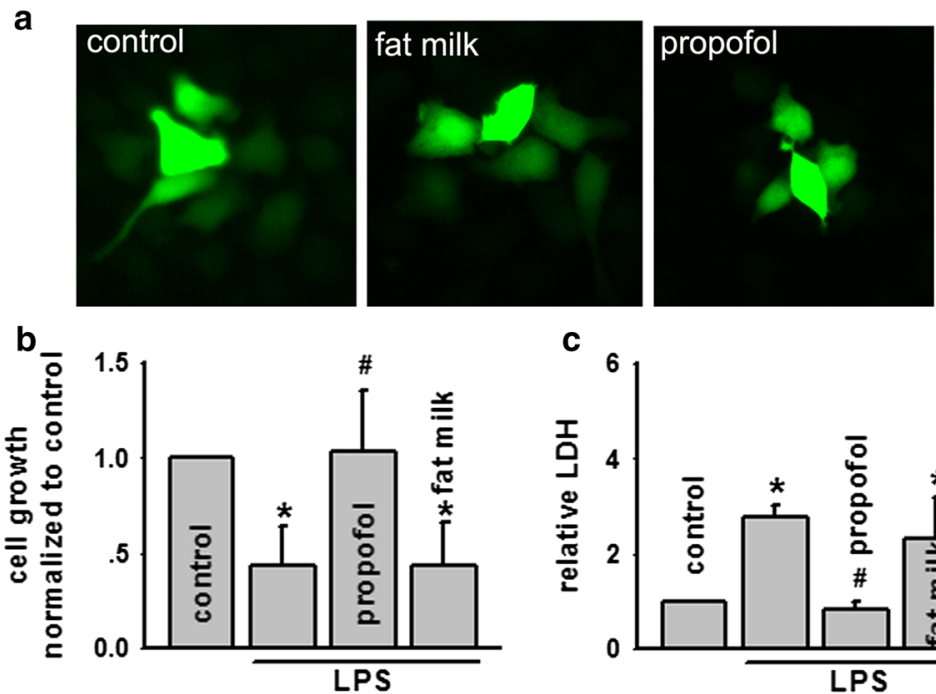

e

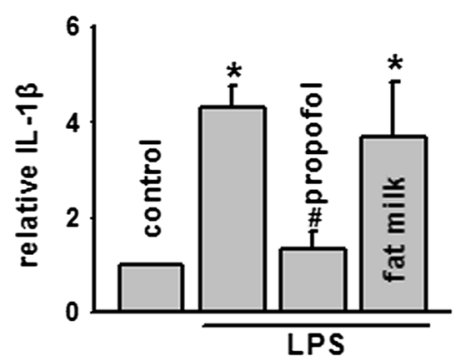

c
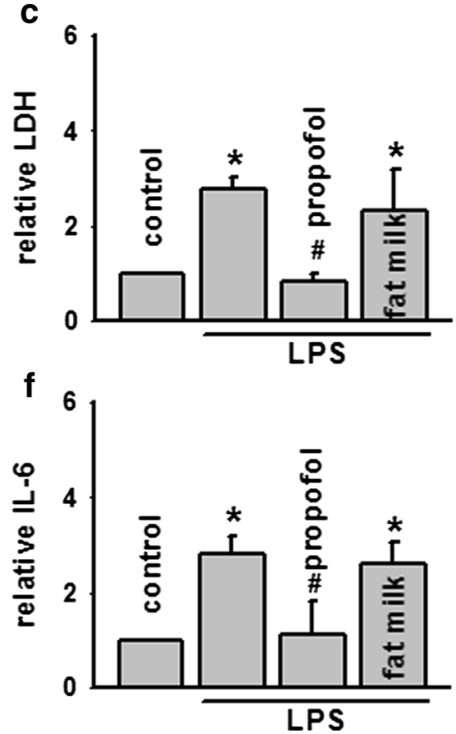

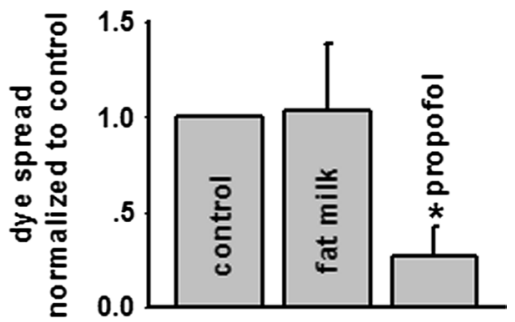

d
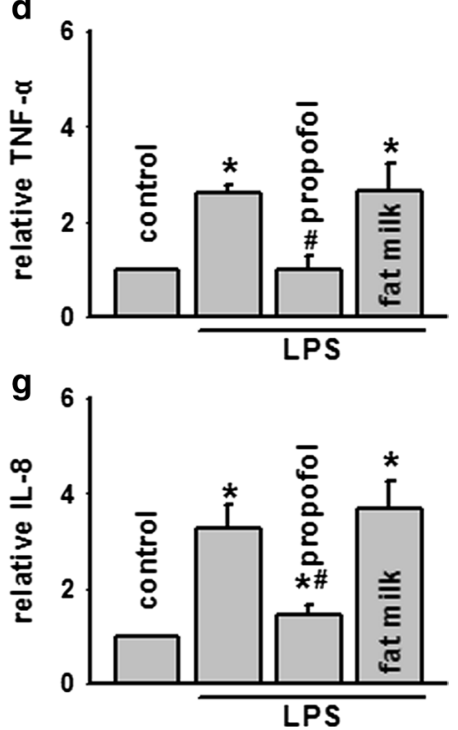

Fig. 5 LPS-induced BEAS-2B cells damage was attenuated by propofol. a "Parachute" dye-coupling assay was used to determine effects of propofol on GJ composed of Cx43. b, c Effects of propofol (15 $\mu \mathrm{M}$, for $1 \mathrm{~h})$ on cell growth and LDH release exposed to LPS (5 $\mu \mathrm{g} / \mathrm{ml}$, for $24 \mathrm{~h}), \mathrm{n}=5,{ }^{*} \mathrm{P}<0.05$

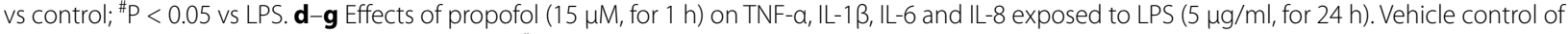
propofol was fat milk, $n=5$, *P $<0.05$ vs control; ${ }^{\#} P<0.05$ vs LPS

between neighboring cells always exerted different effects, the most important one of which was just "death signal", not only directly damaged the neighboring cells, but also activated different signal pathways, resulting in cytotoxicity or apoptosis indirectly $[37,38]$. Through this kind of effect, organ damage was amplified significantly, which was called "bystander effect" [39, 40]. Thus, we supposed that "death signal" transfer interruption was just the reason why GJ composed of Cx43 inhibition could protect against liver transplantation-induced ALI effectively. Pity that the intrinsic quality of "death signal" had not been identified. In our previous study, we had explored the possibility of reactive oxygen species (ROS) as "death signal", but the direct proof was still limited [9]. Although others discussed the possibility of calcium, cells metabolites or molecules, the results were also not satisfied.

In perioperative or preoperative period, proper anesthetics choice was beneficial for patients receiving organ transplantation, because they had protective effects on different organs. We had demonstrated that propofol application, could alleviate rat AOLT-induced ALI, however, the in depth mechanism was still largely unclear [5]; in another investigation, we had clarified that propofol attenuated acute kidney injury via inhibiting GJ composed of Cx32, which indicated GJ inhibition might govern the mechanistic effects of propofol [9]. It had been explored for many years that propofol was always considered to provide protective effects through different mechanisms, such as anti-inflammatory anti-oxidant, or apoptosis inhibition [26, 41, 42]. Incorporating with these facts above, rats were pretreated with propofol to observe its effects on AOLT-induced ALI. Results indicated that propofol alleviated LPS-mediated cell damage through GJ function inhibition in vitro and protected against AOLT-induced ALI also through down-regulating Cx43 GJ function, mechanisms of which were both relative with inflammatory reaction modulation, such as TNF- $\alpha$, IL-1 $\beta$, IL- 6 and IL- 8 . Anti-inflammatory effect of propofol had been investigated for many years, however, mechanisms of which were still not understood very well. As far as we known, signals transfer between 


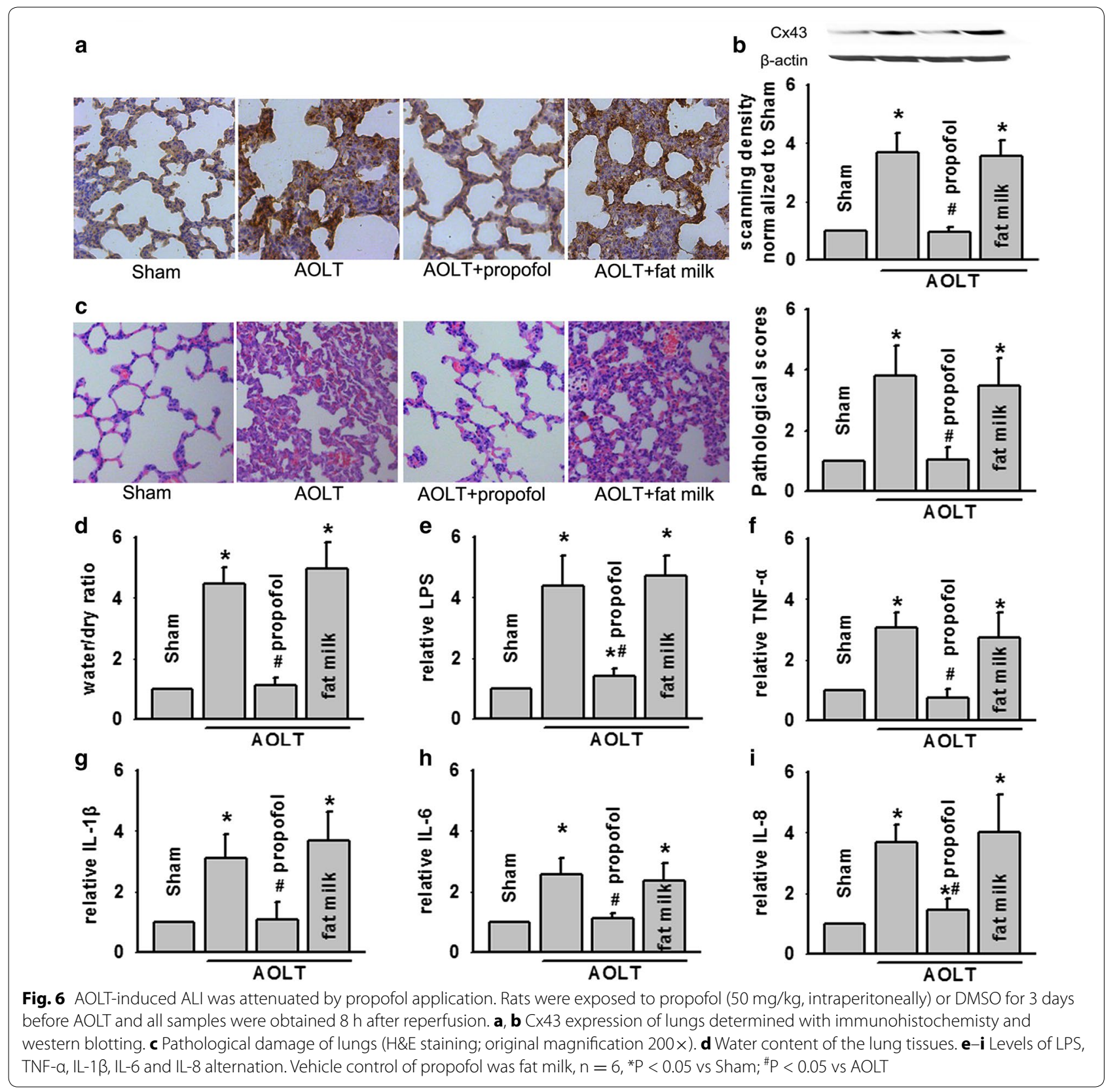

the neighboring cells were less than $1 \mathrm{kDa}$, thus, TNF- $\alpha$, IL-1 $\beta$, IL- 6 and IL-8 (molecular weights were all more than $1 \mathrm{kDa}$ ) could not be transferred through GJ directly. Thus, we supposed that "death signal" was over-produced in this process and transferred in the neighboring cells, triggering or amplifying inflammation reaction significantly, which lead to ALI becoming more and more serious. Hence one can see that $\mathrm{Cx} 43$ inhibition attenuating inflammation reaction was a potential strategy to protect against liver transplantation-induced ALI. Inhibitory effects of propofol on GJ was obvious in Fig. 5. Thus, we believed that protective effects of propofol on AOLTinduced ALI was relative, or at least partially with function of GJ inhibition. We explained anti-inflammatory effect of propofol from another aspect and provided a valid therapeutic target for liver transplantation-induced organ damage, not just only for lungs.

\section{Conclusion}

Liver transplantation was a serious attack for patients and influenced all of the organs and systems. During liver transplantation, because of inferior vena cava and the 
portal vein interruption, intestinal congestion resulted in intestine motility and barriers destroyed obviously. Bacterial translocation and enterogenous endotoxin were over-produced and attacked susceptible organs firstly, not just only for lungs [43, 44]. Our present study mainly demonstrated that propofol application protected against AOLT-induced ALI through Cx43 function inhibition, mechanisms of which were relative with inflammatory reaction decrease. These findings might not be universal to all the remote organs, but at least, this conclusion offered a new basis of targeted intervention for organ protection in liver transplantation, even in other kinds of operation.

\begin{abstract}
Abbreviations
ALl: acute lung injury; Cx43: connexin43; GJ: gap junction; AOLT: autologous orthotopic liver transplantation; LPS: lipopolysaccharide; ARDS: acute respiratory distress syndrome; SVC: supra hepatic vena cava; IVC: inferior vena cava; PV: portal vein; TNF-a: tumor necrosis factor- $\alpha$; LL-1 $\beta$ : interleukin-1 $\beta$; IL-6: inter leukin-6; IL-8: interleukin-8; RA: retinoic acid; CCK-8: cell counting kit-8; siRNA: small interfering RNA; ROS: reactive oxygen species.
\end{abstract}

\section{Authors' contributions}

DY, JC, GL and ZH contributed to the experimental design. DY wrote the manuscript. DY, GS and YL performed the experiments in vivo. DY, XC, JF and QZ performed experiments in vitro. All authors read and approved the final manuscript.

\section{Competing interests}

The authors declared that they have no competing interests.

\section{Ethics approval and consent to participate}

The study was approved by the Laboratory Animal Care Committee of Sun Yat-Sen University (Guangzhou, Guangdong, China) and animal care followed National Institutes of Health criteria for the care and use of laboratory animals in research.

\section{Funding}

This study is supported by the National Natural Science Foundation of China (Beijing, China; Grant nos. 81170449,81401628 and 30972858); Guangzhou Science and Technology Plan (Guangzhou, Guangdong Province, China; Grant nos. 201508030003 and 201607010233); Medical Research Foundation of Guangdong Province (Guangzhou, Guangdong Province, China; Grant no. B2014141).

Received: 25 April 2016 Accepted: 21 June 2016

Published online: 30 June 2016

\section{References}

1. Huang CK, Lee SO, Lai KP, Ma WL, Lin TH, Tsai MY, Luo J, Chang C. Targeting androgen receptor in bone marrow mesenchymal stem cells leads to better transplantation therapy efficacy in liver cirrhosis. Hepatology. 2013;57:1550-63.

2. Hong SK, Hwang S, Lee SG, Lee LS, Ahn CS, Kim KH, Moon DB, Ha TY. Pulmonary complications following adult liver transplantation. Transplant Proc. 2006:38:2979-81.

3. Hirsch J, Niemann CU, Hansen KC, Choi S, Su X, Frank JA, Fang X, Hirose R, Theodore P, Sapru A, et al. Alterations in the proteome of pulmonary alveolar type II cells in the rat after hepatic ischemia-reperfusion. Crit Care Med. 2008;36:1846-54.

4. Huang X, Liu Y, Lu Y, Ma C. Anti-inflammatory effects of eugenol on lipopolysaccharide-induced inflammatory reaction in acute lung injury via regulating inflammation and redox status. Int Immunopharmacol. 2015;26:265-71.

5. Luo G, Zhu G, Yuan D, Yao W, Chi X, Hei Z. Propofol alleviates acute lung injury following orthotopic autologous liver transplantation in rats via inhibition of the NADPH oxidase pathway. Mol Med Rep. 2015;11:2348-54.

6. Zhang A, Chi X, Luo G, Hei Z, Xia H, Luo C, Wang Y, Mao X, Xia Z. Mast cell stabilization alleviates acute lung injury after orthotopic autologous liver transplantation in rats by downregulating inflammation. PLoS One. 2013;8:e75262.

7. Chacko B, Peter JV, Tharyan P, John G, Jeyaseelan L. Pressure-controlled versus volume-controlled ventilation for acute respiratory failure due to acute lung injury (ALI) or acute respiratory distress syndrome (ARDS). Cochrane Database Syst Rev. 2015;1:008807.

8. Hong X, Wang Q, Yang Y, Zheng S, Tong X, Zhang S, Tao L, Harris AL. Gap junctions propagate opposite effects in normal and tumor testicular cells in response to cisplatin. Cancer Lett. 2012;317:165-71.

9. Ge M, Yao W, Wang Y, Yuan D, Chi X, Luo G, Hei Z. Propofol alleviates liver oxidative stress via activating Nrf2 pathway. J Surg Res. 2015;196:373-81.

10. Vinken M, Decrock E, Vanhaecke T, Leybaert L, Rogiers V. Connexin43 signaling contributes to spontaneous apoptosis in cultures of primary hepatocytes. Toxicol Sci. 2012;125:175-86.

11. Gielen PR, Aftab Q, Ma N, Chen VC, Hong X, Lozinsky S, Naus CC, Sin WC. Connexin43 confers Temozolomide resistance in human glioma cells by modulating the mitochondrial apoptosis pathway. Neuropharmacology. 2013;75:539-48.

12. Parthasarathi K. Endothelial connexin 43 mediates acid-induced increases in pulmonary microvascular permeability. Am J Physiol Lung Cell Mol Physiol. 2012;303:L33-42.

13. Parthasarathi K, Ichimura H, Monma E, Lindert J, Quadri S, Issekutz A, Bhattacharya J. Connexin 43 mediates spread of $\mathrm{Ca} 2+$-dependent proinflammatory responses in lung capillaries. J Clin Invest. 2006;116:2193-200.

14. Li X, Li X, Chi X, Luo G, Yuan D, Sun G, Hei Z. Ulinastatin ameliorates acute kidney injury following liver transplantation in rats and humans. Exp Ther Med. 2015;9:411-6.

15. Luo C, Yuan D, Zhao W, Chen H, Luo G, Su G, Hei Z. Sevoflurane ameliorates intestinal ischemia-reperfusion-induced lung injury by inhibiting the synergistic action between mast cell activation and oxidative stress. Mol Med Rep. 2015;12:1082-90.

16. Wang W, Lu R, Feng DY, Liang LR, Liu B, Zhang H. Inhibition of microglial activation contributes to propofol-induced protection against postcardiac arrest brain injury in rats. J Neurochem. 2015;134(5):892-903.

17. Zhao Y, Liu B, Wang Q, Yuan D, Yang Y, Hong X, Wang X, Tao L. Propofol depresses the cytotoxicity of $X$-ray irradiation through inhibition of gap junctions. Anesth Analg. 2011;112:1088-95.

18. Liu KX, Chen SQ, Huang WQ, Li YS, Irwin MG, Xia Z. Propofol pretreatment reduces ceramide production and attenuates intestinal mucosal apoptosis induced by intestinal ischemia/reperfusion in rats. Anesth Analg. 2008;107:1884-91.

19. Franco-Gou R, Rosello-Catafau J, Peralta C. Protection against lung damage in reduced-size liver transplantation. Crit Care Med. 2006;34:1506-13.

20. Luo C, Yuan D, Li X, Yao W, Luo G, Chi X, Li H, Irwin MG, Xia Z, Hei Z. Propofol attenuated acute kidney injury after orthotopic liver transplantation via inhibiting gap junction composed of connexin 32. Anesthesiology. 2015;122:72-86.

21. Yuan D, Wang Q, Wu D, Yu M, Zhang S, Li L, Tao L, Harris AL. Monocyteendothelial adhesion is modulated by CX43-stimulated ATP release from monocytes. Biochem Biophys Res Commun. 2012;420:536-41.

22. Li K, Chi Y, Gao K, Yan Q, Matsue H, Takeda M, Kitamura M, Yao J. Connexin43 hemichannel-mediated regulation of connexin43. PLoS One. 2013;8:e58057.

23. Wang Y, Rangan GK, Goodwin B, Tay YC, Harris DC. Lipopolysaccharideinduced MCP-1 gene expression in rat tubular epithelial cells is nuclear factor-kappaB dependent. Kidney Int. 2000;57:2011-22.

24. De Hert SG, ten Broecke PW, Mertens E, Van Sommeren EW, De Blier IG, Stockman BA, Rodrigus IE. Sevoflurane but not propofol preserves myocardial function in coronary surgery patients. Anesthesiology. 2002;97:42-9.

25. De Hert SG, Cromheecke S, ten Broecke PW, Mertens E, De Blier IG, Stockman BA, Rodrigus IE, Van der Linden PJ. Effects of propofol, desflurane, 
and sevoflurane on recovery of myocardial function after coronary surgery in elderly high-risk patients. Anesthesiology. 2003;99:314-23.

26. Zhu M, Wen M, Sun X, Chen W, Chen J, Miao C. Propofol protects against high glucose-induced endothelial apoptosis and dysfunction in human umbilical vein endothelial cells. Anesth Analg. 2015;120:781-9.

27. Gan X, Xing D, Su G, Li S, Luo C, Irwin MG, Xia Z, Li H, Hei Z. Propofol attenuates small intestinal ischemia reperfusion injury through inhibiting NADPH oxidase mediated mast cell activation. Oxid Med Cell Longev. 2015;2015:167014.

28. Ge M, Luo G, Yao W, Luo C, Zhou S, Yuan D, Chi X, Hei Z. Propofol pretreatment attenuates remote kidney injury induced by orthotopic liver autotransplantation, which is correlated with the activation of Nrf2 in rats. Mol Med Rep. 2015;11:3962-8.

29. Tianzhu Z, Shumin W. Esculin inhibits the inflammation of Ips-induced acute lung injury in mice via regulation of TLR/NF-kappaB pathways. Inflammation. 2015;38(4):1529-36.

30. Petroni RC, Biselli PJ, de Lima TM, Theobaldo MC, Caldini ET, Pimentel RN, Barbeiro HV, Kubo SA, Velasco IT, Soriano FG. Hypertonic saline ( $\mathrm{NaCl}$ $7.5 \%$ ) reduces LPS-induced acute lung injury in rats. Inflammation. 2015:38(6):2026-35.

31. Mueller AR, Platz KP, Kremer B. Early postoperative complications following liver transplantation. Best Pract Res Clin Gastroenterol. 2004;18:881-900

32. Yi HM, Cai CJ, Lu MQ, Wang GS, Yi SH, Yang Y, Xu C, Li H, Chen GH. The treatment strategy of early ALI after liver transplantation. Zhonghua Wai Ke Za Zhi. 2006:44:889-93.

33. Goto S, Kamada N, Moore T, Ware F, Lord R, Kobayashi E, Kim YI. The influence of intestinal congestion on survival of preserved liver grafts in rat liver transplantation. Transplantation. 1994:58:974-7.

34. Sanada Y, Urahashi T, Ihara Y, Okada N, Yamada N, Hirata Y, Mizuta K. Pretransplant levels of endotoxin can predict the risk of bacterial infections and graft liver function after liver transplantation. Eur J Pediatr Surg. 2015:25:236-41.

35. Pinali C, Bennett HJ, Davenport JB, Caldwell JL, Starborg T, Trafford AW, Kitmitto A. Three-dimensional structure of the intercalated disc reveals plicate domain and gap junction remodeling in heart failure. Biophys $\mathrm{J}$. 2015;108:498-507.

36. Shin SY, Jo WM, Min TJ, Kim BK, Song DH, Hyeon SH, Kwon JE, Lee WS, Lee KJ, Kim SW, et al. Gap junction remodelling by chronic pressure overload is related to the increased susceptibility to atrial fibrillation in rat heart. Europace. 2015;17:655-63.

37. Krutovskikh VA, Piccoli C, Yamasaki H. Gap junction intercellular communication propagates cell death in cancerous cells. Oncogene. 2002:21:1989-99.

38. Le HT, Sin WC, Lozinsky S, Bechberger J, Vega JL, Guo XQ, Saez JC, Naus CC. Gap junction intercellular communication mediated by connexin43 in astrocytes is essential for their resistance to oxidative stress. J Biol Chem. 2014;289:1345-54

39. Liu Z, Wang Q, Fan L, Wu DP, Zhang Y, Liu L, Tao L. Gap junction enhances phototoxicity of photodynamic therapy agent 2- [1-hexyloxyethyl]2-devinylpyropheophorbide-a (HPPH). Lasers Surg Med. 2015;47:68-76.

40. Akopian A, Atlasz T, Pan F, Wong S, Zhang Y, Volgyi B, Paul DL, Bloomfield SA. Gap junction-mediated death of retinal neurons is connexin and insult specific: a potential target for neuroprotection. J Neurosci. 2014:34:10582-91.

41. Bertolizio G, Ingelmo P. In reply: propofol and additives in studies evaluating inflammatory responses. J Pediatr Hematol Oncol. 2015;37:73-4.

42. Erbas M, Demiraran Y, Yildirim HA, Sezen G, Iskender A, Karagoz I, Kandis H. Comparison of effects on the oxidant/antioxidant system of sevoflurane, desflurane and propofol infusion during general anesthesia. Rev Bras Anestesiol. 2015;65:68-72.

43. Sanada Y, Mizuta K, Urahashi T, Ihara Y, Wakiya T, Okada N, Yamada N, Ushijima K, Otomo S, Sakamoto K, et al. Impact of endotoxin measured by an endotoxin activity assay during liver transplantation. J Surg Res. 2013;180:349-55.

44. Tange S, Hofer Y, Welte M, Anthuber M, Jauch KW, Geissler EK, Ertel W. Local secretion of TNF-alpha from the liver does not correlate with endotoxin, IL-6, or organ function in the early phase after orthotopic liver transplantation. Transpl Int. 2001;14:80-6.

\section{Submit your next manuscript to BioMed Central and we will help you at every step:}

- We accept pre-submission inquiries

- Our selector tool helps you to find the most relevant journal

- We provide round the clock customer support

- Convenient online submission

- Thorough peer review

- Inclusion in PubMed and all major indexing services

- Maximum visibility for your research

Submit your manuscript at www.biomedcentral.com/submit
O Biomed Central 